\title{
FIRST RESULTS OF THE ABSOLUTE CALIBRATION OF THE $u, v, b, y$ SYSTEM OF FILTERS
}

\author{
S. TAPIA and H. L. JOHNSON \\ Steward Observatory, University of Arizona, Tucson, Ariz., U.S.A. \\ and \\ D. L. CRAWFORD \\ Kitt Peak National Observatory*, Tucson, Ariz., U.S.A.
}

\begin{abstract}
Absolute flux densities for a zero magnitude AOV star at the effective wavelengths of the $u, v, b, y$ spectral bands are presented.
\end{abstract}

King (1952) has shown that a magnitude obtained in a given spectral band behaves, to a first approximation, like monochromatic magnitude at an effective wavelength given by

$$
\lambda_{\text {eff }}=\frac{\int_{0}^{\infty} \lambda \phi(\lambda) \mathrm{d} \lambda}{\int_{0}^{\infty} \phi(\lambda) \mathrm{d} \lambda},
$$

where $\phi(\lambda)$ is the sensitivity of the measuring instrument defining the spectral band. If the absolute flux density of a source is known at the effective wavelength of a given spectral band, the relation between instrumental and monochromatic magnitudes permits the absolute calibration of that spectral band. We have used the relation derived by King (1952) to obtain the absolute calibration of the $u, v, b, y$ system of filters.

Several standard stars and a lamp of calibrated spectral distribution were observed with $u, v, b, y$ filters at the Kitt Peak National Observatory. The observed magnitudes were combined with the relative sensitivity of the photometer to derive absolute flux densities. The first results have been used to test the calibration computing the absolute flux densities of a zero magnitude A $0 \mathrm{~V}$ star at the effective wavelengths of the $u, v$, $b, y$ spectral bands. The computed effective wavelengths and absolute flux densities are given in Table I. A comparison of our results with published absolute flux densities for A0 V stars is presented in Figure 1.

A check of the spectral distribution of the lamp used in the observations is in progress. The final results and directions for use of the calibration will be published elsewhere.

* Operated by the Association of Universities for Research in Astronomy, Inc., under contract with the National Science Foundation. 


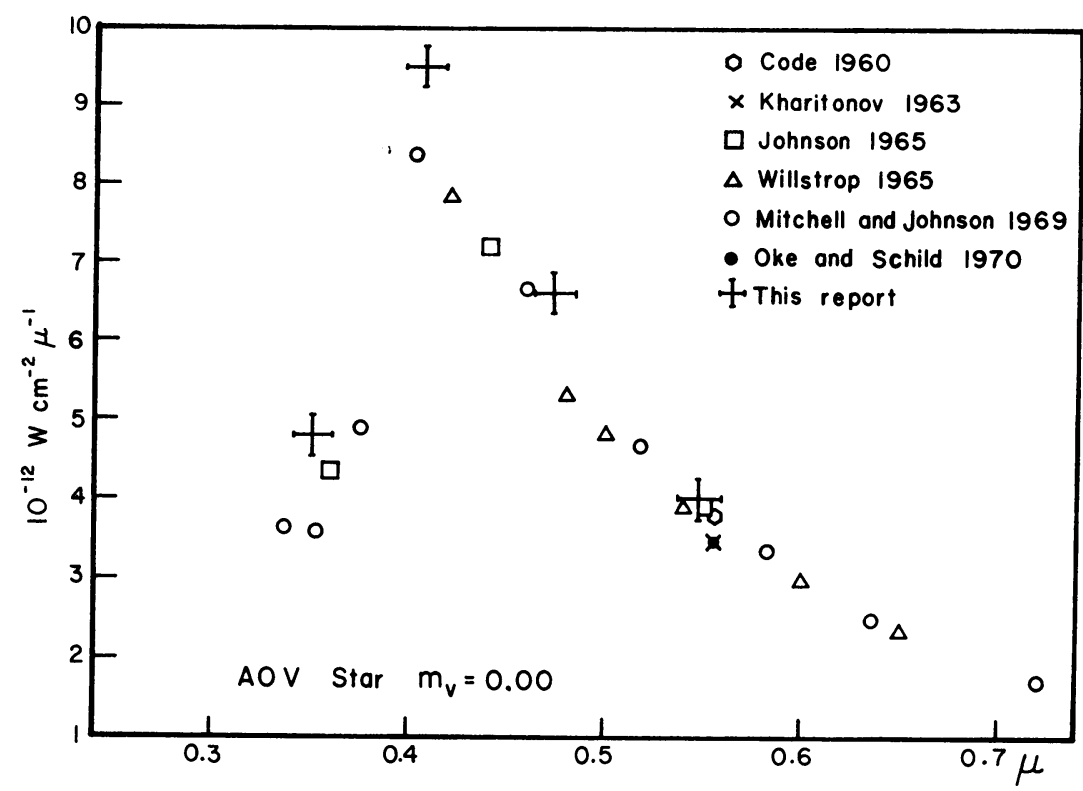

Fig. 1. Absolute flux densities, obtained from different calibrations, for a zero magnitude A0 V star. The abscissae are wavelengths in units of micron and the ordinates are flux densities in units of $10^{-12} \mathrm{~W} \mathrm{~cm}^{-1} \mu^{-1}$. The values of Table I are shown with crosses.

\section{TABLE I}

Absolute calibration of the $u, v, b, y$ system. First results

\begin{tabular}{lll}
\hline Filter & $\lambda_{\text {eff }}$ & $\begin{array}{l}\text { Absolute flux density } \\
\text { (zero mag. A0 V star) }\end{array}$ \\
\hline$u$ & $0.348 \mu$ & $4.82 \times 10^{-12} \mathrm{~W} \mathrm{~cm}^{-2} \mu^{-1}$ \\
$v$ & $0.401 \mu$ & $9.52 \times 10^{-12} \mathrm{~W} \mathrm{~cm}^{-2} \mu^{-1}$ \\
$b$ & $0.462 \mu$ & $6.63 \times 10^{-12} \mathrm{~W} \mathrm{~cm}^{-2} \mu^{-1}$ \\
$y$ & $0.545 \mu$ & $4.00 \times 10^{-12} \mathrm{~W} \mathrm{~cm}^{-2} \mu^{-1}$ \\
\hline
\end{tabular}

\section{References}

Code, A. D.: 1960, Stars and Stellar Systems 6, 85.

Johnson, H. L.: 1965, Commun. Lunar Planet. Lab. 3, No. 53, 73.

Kharitonov, A.: 1963, Soviet Astron. 7, 258.

King, I.: 1952, Astron. J. 57, 253.

Mitchell, R. I. and Johnson, H. L.: 1969, Commun. Lunar Planetary Lab. 8, No. 132, 1.

Oke, J. B. and Schild, R. E.: 1970, Astrophys. J. 161, 1022.

Willstrop, R. V.: 1965, Mem. Roy. Astron. Soc. 69, 83. 